Reprod. Nutr. Dévelop., 1984, 24 (1), 33-44.

\title{
Activité électromyographique de l'utérus chez la brebis pendant la saison sexuelle : comparaison de l'œstrus naturel et de l'œestrus induit par les progestagènes seuls ou avec une supplémentation de PMSG
}

\author{
Marie-Jeanne PRUD'HOMME, B. PELE (') \\ avec la collaboration technique de Nicole LACROIX et G. COUTHERUT
}

Station de Physiologie de la Reproduction, I.N.R.A., Nouzilly, 37380 Monnaie, France.

Summary. Electromyographic activity of the ewe uterus during the breeding season : comparison of natural oestrus and oestrus induced by progestagens with or without PMSG supplementation.

Electromyographic (EMG) activity of the uterus was recorded in vivo in 3 groups of ewes in oestrus during the breeding season. The ewes were either in natural oestrus or in oestrus induced by progestagens with or without PMSG supplementation. Plasma concentrations of $\mathrm{LH}$ were measured at regular intervals in order to determine the onset of the preovulatory surge of LH (To).

During natural oestrus, the uterus exhibited a spontaneous rhythmic activity composed of bursts of potentials. Burst frequency was maximal at the moment of preovulatory LH release, then decreased. Percentages of downward, upward and non-definite propagations were calculated during 1-h periods at the beginning of the $\mathrm{LH}$ peak and $6,12,24$ and $36 \mathrm{~h}$ afterwards. There was no difference between the relative proportions of these types of propagation at any of the times analysed:

Mean burst frequency was not modified by progestagen pre-treatment but showed more interanimal variability at the moment of maximal frequency in ewes treated with progestagen. For the first $12 \mathrm{~h}$ after To, the organization of uterine activity was not modified by progestagens but non-definite propagation became predominant 24 and $36 \mathrm{~h}$ after To, namely from the time of ovulation onward.

PMSG increased burst frequency. The increase of frequency variability between animals, normally observed at the beginning of oestrus, and the predominance of nondefinite propagation, normally observed at the end of progestagen-induced oestrus, did not appear when the ewes had been treated with PMSG.

\section{Introduction.}

Chez la Brebis, un traitement progestatif est couramment utilisé afín de synchroniser l'œstrus. Dans ces conditions, la fertilité est inférieure à celle observée après l'œstrus naturel (Colas et al., 1973). Lorsqu'une administration d'hor-

(1) Adresse actuelle : Institut de Sélection Animale, Le Fœil, 22800 Quintin, France. 
mone gonadotrope (PMSG) est associée à ce traitement, la fertilité est identique à ce qu'elle est dans les conditions naturelles (Colas, 1975). La diminution de fertilité constatée dans le premier cas a été attribuée à un transit anormal et/ou à une réduction de la possibilité de survie des spermatozoïdes dans les voies génitales femelles (Quinlivan et Robinson, 1967, 1969 ; Hawk et Conley, 1971, 1972). L'un des facteurs contribuant au transit des spermatozoïdes est la motricité utérine (Mattner, 1963). On peut supposer que le contrôle de l'œstrus par les progestagènes seuls conduit à une altération de cette motricité et à une perturbation du transit des spermatozoïdes. La PMSG préviendrait cette dernière. Le but de notre étude a donc été de comparer, pendant la saison sexuelle, la motricité utérine de la Brebis au cours de l'œstrus naturel et au cours de l'œestrus induit par les progestagènes, les animaux étant dans ce dernier cas, traités ou non par la PMSG.

\section{Matériel et méthodes.}

Les expériences sont réalisées pendant la saison sexuelle sur 23 brebis cycliques de race Préalpes du Sud. 8 animaux sont utilisés au cours de l'œestrus naturel (Lot $N$ ), 8 au cours de l'œstrus induit par les progestagènes seuls (Lot $I_{1}$ ) et 7 au cours de l'œstrus induit par les progestagènes avec une supplémentation de PMSG (Lot $\mathrm{I}_{2}$ ). L'cestrus est induit à l'aide d'éponges vaginales imprégnées de $40 \mathrm{mg}$ d'acétate de fluorogestone (FGA) (chrono-gest éponges-Intervet) maintenues en place pendant 14 jours (Lot $I_{1}$ ). Pour le lot $I_{2}, 400$ UI d'hormone gonadotrope extraite de sérum de jument gravide (PMSG) (chrono-gest PMSG-Intervet) sont injectées par voie intramusculaire au moment du retrait des éponges.

La détection de l'œstrus est effectuée par la présentation du bélier aux brebis du lot $N$, toutes les $2 h$, du $16^{e}$ jour du cycle jusqu'à l'apparition des premières manifestations comportementales. Pour avoir un repère précis de l'état physiologique des animaux, les concentrations plasmatiques de $\mathrm{LH}$ sont mesurées selon la technique de Pelletier et al. (1968), à partir d'échantillons de sang prélevé dans la veine jugulaire toutes les $2 \mathrm{~h}$. Les prélèvements sont réalisés le $16^{\mathrm{e}}$ jour du cycle jusqu'à $24 \mathrm{~h}$ après le début des chaleurs pour le lot $N$, de 24 à $72 \mathrm{~h}$ après le retrait des éponges pour les lots $I_{1}$ et $I_{2}$. La courbe des concentrations plasmatiques de $\mathrm{LH}$ en fonction du temps permet de repérer le début du pic ou temps To. II correspond au dernier point bas de la courbe $(<10 \mathrm{ng} / \mathrm{ml})$, avant l'augmentation de la concentration.

L'activité électromyographique (EMG) du myomètre est recueillie en 3 points d'une corne utérine par des électrodes bipolaires de platine placées à demeure selon la technique de Rousseau et Prud'homme (1974). Les variations de potentiel sont amplifiées et enregistrées sur papier grâce à un inscripteur à plumes classique (Beckman). Pour les brebis en cestrus naturel, l'enregistrement commence le $16^{\mathrm{e}}$ jour du cycle et se poursuit jusqu'au $2^{\mathrm{e}}$ jour du cycle suivant. Pour les brebis en œstrus induit, l'enregistrement commence $24 \mathrm{~h}$ après la dépose de l'éponge et dure $72 \mathrm{~h}$. Deux vitesses de déroulement du papier sont utilisées ; $50 \mathrm{~mm} / \mathrm{min}$ et $5 \mathrm{~mm} / \mathrm{s}$.

L'activité EMG du myomètre est quantifiée, pour chaque brebis par la fréquence des salves de potentiels : le nombre de salves est compté par tranches de 
10 min à partir de $10 \mathrm{~h}$ avant le début du pic de LH (temps To) jusqu'à 30 à $40 \mathrm{~h}$ après, cette période incluant l'œestrus. Par le tracé dune courbe individuelle de régression au $5^{\mathrm{e}}$ degré représentant le nombre de salves par $10 \mathrm{~min}$ en fonction du temps, on détermine par rapport au temps To le moment où la fréquence des salves est maximale (Tf. max.), chez chaque brebis. Les variations de la fréquence des salves, pour un lot de brebis, sont représentées de 2 façons selon l'origine des temps choisie. La fréquence moyenne des salves, exprimée en nombre de salves par 10 min, est calculée pour chaque brebis d'un lot sur sept périodes successives de $6 \mathrm{~h}$ d'enregistrement, une période avant et 6 périodes après le début du pic de LH. Elle est également calculée pour la période de $6 \mathrm{~h}$ qui encadre le Tf. max., ainsi que pour 6 autres tranches de $6 \mathrm{~h}$, l'une précédant la période correspondant au Tf. max., les 5 autres la suivant. Les moyennes des fréquences, pour chaque période, permettent de construire deux courbes moyennes par lot, l'une ayant comme origine des temps le début du pic de LH, l'autre le Tf. max. Les courbes moyennes de chacun des 3 lots de brebis sont comparées en utilisant les tests $t$ et F.

Les enregistrements à grande vitesse $(5 \mathrm{~mm} / \mathrm{s})$ sont effectués par périodes d'une heure à intervalles réguliers $(0,6,12,24$ et $36 \mathrm{~h}$ après le début du pic de LH). Ils permettent d'analyser l'organisation de la motricité utérine. Nous notons pour chaque brebis et pour chaque période, le pourcentage de salves qui apparaissent successivement sous les 3 électrodes dans le sens jonction utéro-tubaire (JUT)-cervix (propagation descendante), dans le sens cervix-JUT (propagation ascendante) et celui des salves dont l'ordre d'apparition sous les électrodes ne permet pas de caractériser l'un de ces deux sens (propagation non définie). Pour chaque lot et pour chaque période, les pourcentages des 3 types de propagation de chaque brebis sont affectés des rangs 1, 2 ou 3 selon leur classement. Les tests de Friedman et de Kramer sur la somme des rangs à l'intérieur de chaque lot sont utilisés afin de rechercher les différences significatives entre ces 3 pourcentages.

\section{Résultats.}

1. - Description de l'activité EMG du myomètre. - Pour les 3 lots de brebis, il apparaît, 18 à 24 h avant le début du pic de LH, une activité rythmique spontanée caractérisée par des salves de potentiels (fig. 1A). Ces salves, d'une durée de

A

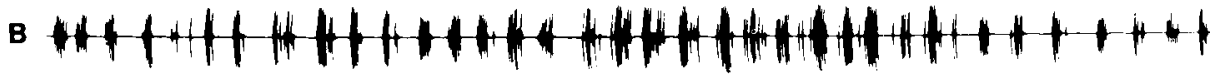

C

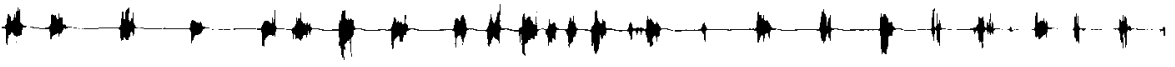

FIG. 1. - Enregistrement de l'activité électrique du myomètre chez une brebis cyclique. A : $20 \mathrm{~h}$ avant le début du pic de LH ; B : au début du pic de $\mathrm{LH} ; \mathrm{C}: 20 \mathrm{~h}$ après le début du pic de $\mathrm{LH}$. La fréquence des salves et l'amplitude des potentiels qui les composent sont augmentées au moment du pic de LH chez cet animal.

Etalonnages : Amplitude : $500 \mu \mathrm{V}(\mathrm{A}) ; 1 \mathrm{mV}$ (B et C) ; Temps : $10 \mathrm{~s}$. 

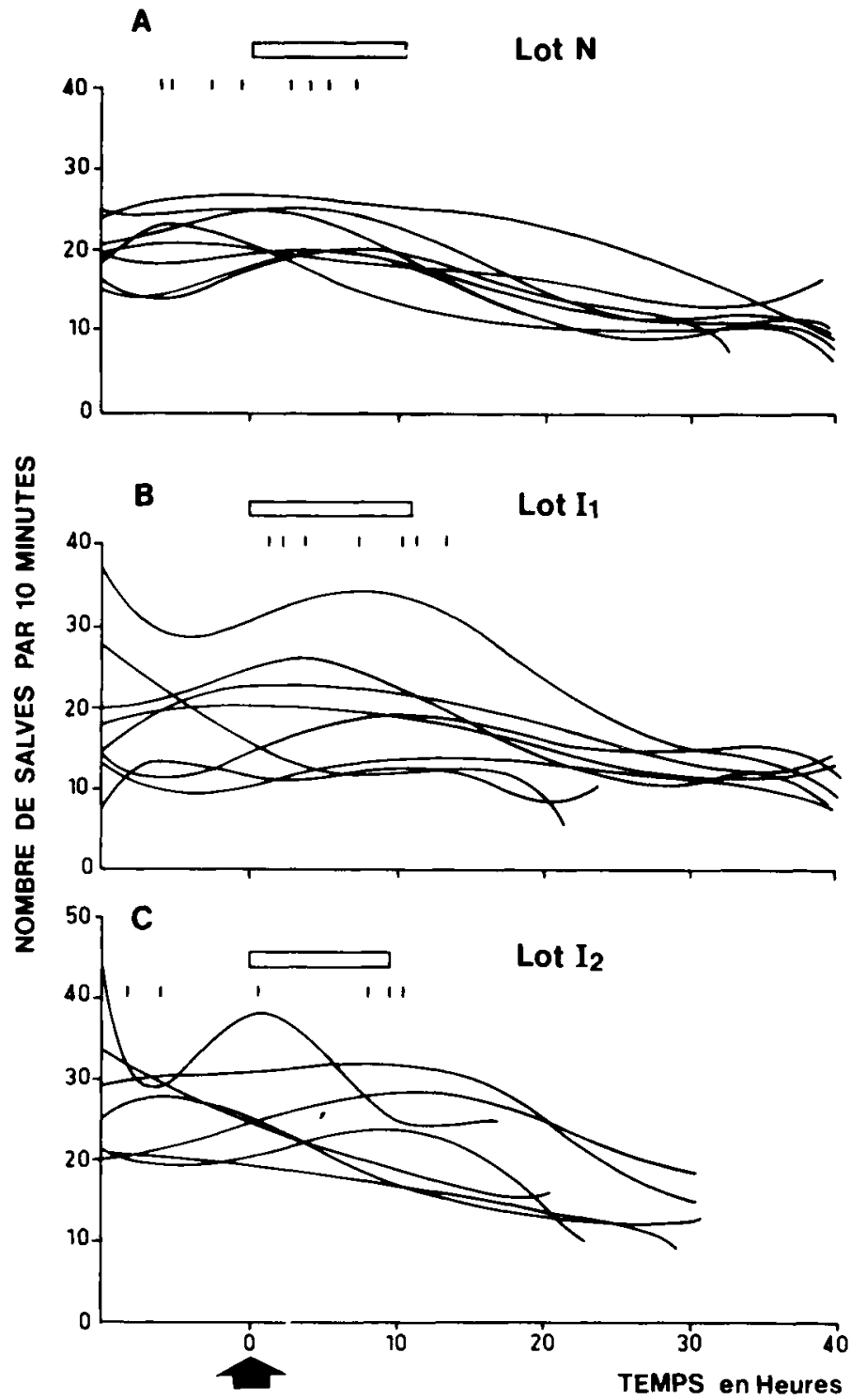

Début du pic de LH

FIG. 2. - Evolution de la fréquence des salves de potentiels chez 3 lots de brebis cycliques. $A$ : lot $N$, au cours de I'œestrus naturel ; $B$ : lot $\mathrm{I}_{1}$, au cours de l'œestrus induit par les progestagènes seuls ; $C$ : lot $I_{2}$, au cours de l'œestrus induit par les progestagènes avec une supplémentation de PMSG.

Abcisses : temps en heures par rapport au début du pic de LH ; Ordonnées : nombre de salves par $10 \mathrm{~min}$.

Les tracés sont les courbes de régression au $5^{e}$ degré représentant pour chaque brebis le nombre de salves comptées par $10 \mathrm{~min}$ en fonction du temps. Pour chaque lot, la durée moyenne de la décharge de LH est représentée par la barre horizontale. Pour chaque courbe, le moment de la fréquence maximale est indiqué par un trait vertical. 
2 à $10 \mathrm{~s}$, sont constituées de 3 à 12 potentiels. Leur fréquence évolue dans le temps comme le montrent les enregistrements $A, B$ et $C$ de la figure 1 . Les courbes de régression obtenues à partir du nombre de salves par 10 min passent par un maximum (fig. 2).

2. - Evolution de la fréquence des salves de potentiels des 3 lots de brebis. - Les fréquences maxima (Tf. max.) se situent de part et d'autre du début du pic de LH (To) : pour le lot $\mathrm{N}$, ils se répartissent sur une période de $13 \mathrm{~h}$ entre -6 et $+7 \mathrm{~h}$ (fig. 2A). Pour le lot $\mathrm{l}_{1}$, ils se situent entre -20 et $+14 \mathrm{~h}$. Cependant, 7 maxima sur 8 (soit $88 \%$ ) sont regroupés sur une période de $13 \mathrm{~h}$ entre +1 et $+14 \mathrm{~h}$ (fig. 2B). Pour le lot $\mathrm{I}_{2}$, ils se répartissent sur une période de $24 \mathrm{~h}$ entre -13 et +11 h (fig. $2 \mathrm{C}$ ).

En moyenne, pour le lot $\mathrm{N}$, le nombre de salves par 10 min est stable jusqu'à $12 \mathrm{~h}$ après le début du pic de $\mathrm{LH}$ et se situe entre 20,72 et 21,15 . La fréquence chute ensuite jusqu'à 16,80 salves par 10 min puis décroît régulièrement (fig. 3 et

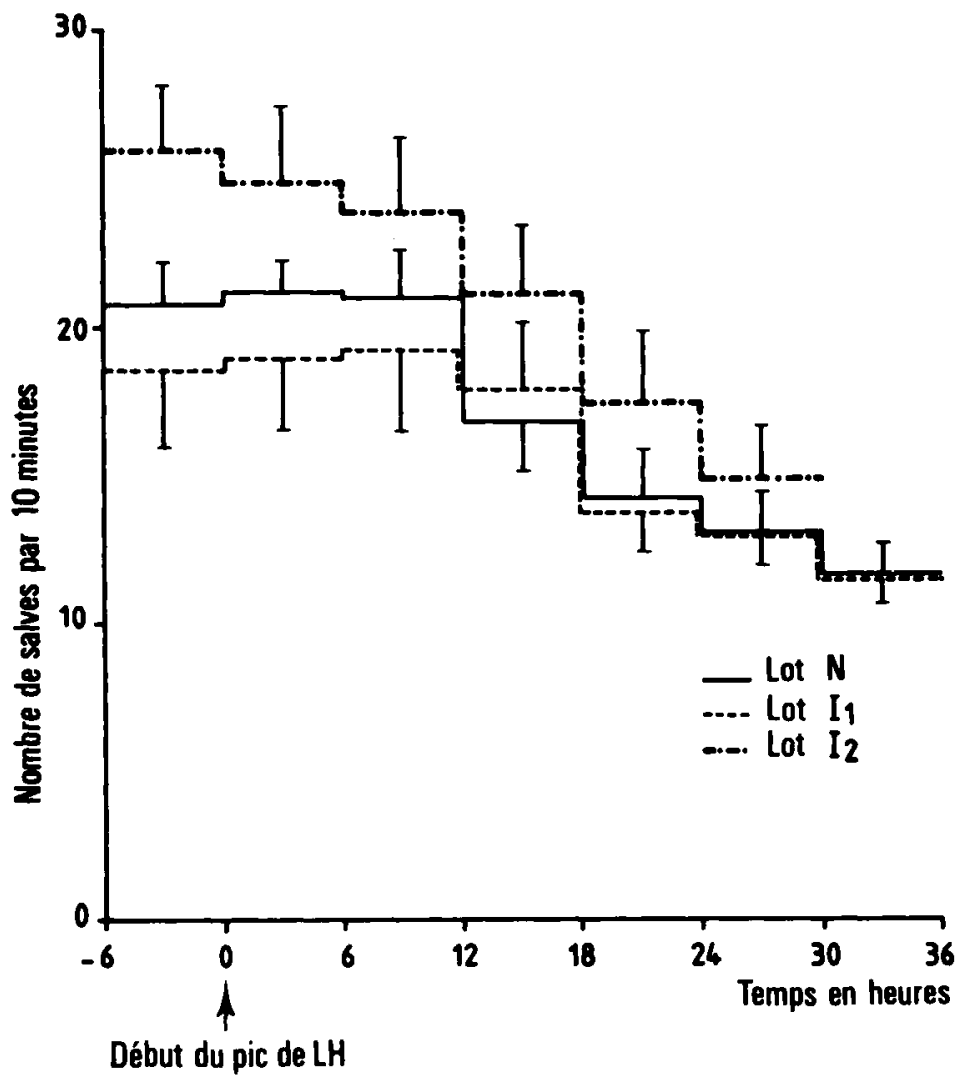

FIG. 3. - Evolution moyenne de la fréquence des salves de potentiels chez 3 lots de brebis. Lot $\mathrm{N}$ : œstrus naturel ; Lot $\mathrm{I}_{1}$ : œestrus induit par les progestagènes ; Lot $\mathrm{I}_{2}$ : œstrus induit par les progestagènes avec une supplémentation de PMSG.

Abcisses : temps en heures par rapport au début du pic de $\mathrm{LH}$; Ordonnées : moyenne \pm sem du nombre de salves par $10 \mathrm{~min}$, déterminée pour chacun des 3 lots par tranches successives de $6 \mathrm{~h}$. 
tabl. 1). Pour le lot $\mathrm{I}_{1}$, la fréquence varie peu jusqu'à $+18 \mathrm{~h}$ et se situe entre 17,88 et 19,23 salves par $10 \mathrm{~min}$. Elle chute ensuite jusqu'à 13,71 salves par $10 \mathrm{~min}$ puis décroît régulièrement (fig. 3 et tabl. 1). Pour chacune des tranches de $6 \mathrm{~h}$, les moyennes du lot $I_{1}$ ne sont pas différentes de celles du lot $N$ $\left(P>0,05\right.$, tabl. 1). Dans la tranche $(0,+6)$, la variance du lot $l_{1}$ est supérieure à celle du lot $N\left(P<0,05\right.$, tabl. 1). Pour le lot $I_{2}$, le nombre de salves par $10 \mathrm{~min}$ décroît légèrement de 26,02 à 23,92 jusqu'à $12 \mathrm{~h}$ après le début du pic de $\mathrm{LH}$; la diminution est ensuite plus rapide (fig. 3 et tabl. 1). Pour chacune des tranches de $6 \mathrm{~h}$, les moyennes et les variances du lot $\mathrm{I}_{2}$ ne sont pas différentes de celles du lot $N(P>0,05$, tabl. 1$)$.

\section{TABLEAU 1}

Evolution en fonction du temps, par rapport au début du pic de $\mathrm{LH}$, de la fréquence moyenne des salves par 10 min chez les 3 lots de brebis.

\begin{tabular}{|c|c|c|c|}
\hline \multirow{2}{*}{$\begin{array}{c}\text { Temps (h) } \\
\text { par rapport } \\
\text { au début du pic de LH (To) }\end{array}$} & \multicolumn{3}{|c|}{ Moyenne \pm s.e.m. du nombre de salves par $10 \mathrm{~min}$} \\
\hline & Lot $\mathrm{N}$ & Lot $I_{1}$ & Lot $I_{2}$ \\
\hline 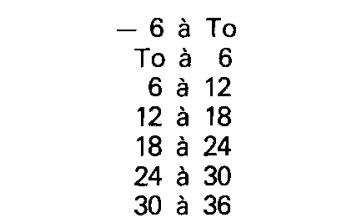 & $\begin{array}{l}20,72 \pm 1,48 \\
21,15 \pm 0,97 a \\
20,91 \pm 1,53 \\
16,80 \pm 1,73 \\
14,27 \pm 1,46 \\
13,07 \pm 1,24 \\
11,67 \pm 0,75\end{array}$ & $\begin{array}{l}18,57 \pm 2,71 \\
19,01 \pm 2,51 \mathrm{a} \\
19,23 \pm 2,70 \\
17,88 \pm 2,16 \\
13,71 \pm 1,34 \\
13,01 \pm 1,16 \\
11,43 \pm 0,79\end{array}$ & $\begin{array}{l}26,02 \pm 2,25 \\
24,89 \pm 2,40 \\
23,92 \pm 2,10 \\
21,10 \pm 2,18 \\
17,37 \pm 2,38 \\
14,75 \pm 1,69\end{array}$ \\
\hline
\end{tabular}

a : dans l'intervalle $(T o,+6)$ la variance du lot $I_{1}$ est supérieure à celle du lot $N(P<0,05)$.

TABLEAU 2

Evolution en fonction du temps, par rapport au moment de la fréquence maximale, de la fréquence moyenne des salves par 10 min chez les 3 lots de brebis.

\begin{tabular}{|c|c|c|c|}
\hline \multirow{2}{*}{$\begin{array}{l}\text { Temps (h) par rapport } \\
\text { au moment de la fréquence } \\
\text { maximale (Tf. max.) }\end{array}$} & \multicolumn{3}{|c|}{ Moyenne \pm s.e.m. du nombre de salves par $10 \mathrm{~min}$} \\
\hline & Lot $N$ & Lot $\mathrm{I}_{1}$ & Lot $\mathrm{I}_{2}$ \\
\hline$-9 a-3$ & ${ }^{\mathrm{b}} 20,74 \pm 1,25^{\mathrm{a}}$ & $21,39 \pm 3,12^{a}$ & $b_{26,58} \pm 2,83$ \\
\hline-3 à 3 & $\mathrm{~b} 22,92 \pm 1,17^{\mathrm{a}}$ & $22,79 \pm 3,04^{3}$ & b $29,36 \pm 2,11$ \\
\hline 3 à & ${ }^{\circ} 20,44 \pm 1,29 \mathrm{a}$ & $20,57 \pm 2,86^{\circ}$ & $\mathrm{b} 25,25 \pm 1,90$ \\
\hline 9 à & b $16,30 \pm 1,51$ & $17,72 \pm 2,43$ & b $22,24 \pm 2,16$ \\
\hline 15 à 21 & b $14,11 \pm 1,35$ & $16,13 \pm 1,71$ & $\mathrm{~b} 19,75 \pm 2,14$ \\
\hline 21 à 27 & b $12,55 \pm 1,03$ & $12,30 \pm 0,67$ & b $16,22 \pm 1,48$ \\
\hline 27 à 33 & $b 11,30 \pm 0,65$ & $11,23 \pm 0,59$ & $\mathrm{~b} 13,76 \pm 0,87$ \\
\hline
\end{tabular}

a : les variances du lot $I_{1}$ sont supérieures à celles du lot $\mathrm{N}$ dans les 3 premières tranches $(P<0,05)$.

$b$ : les moyennes du lot $\mathrm{I}_{2}$ sont supérieures à celles du lot $\mathrm{N}$ pour chacune des tranches $(P<0,05)$. 
Lorsque l'origine des temps correspond au moment du maximum de fréquence, les similitudes et les différences apparaissent plus nettement. Les courbes moyennes des lots $N$ et $I_{1}$ suivent une évolution identique (fig. 4). Pour chacune des tranches de $6 \mathrm{~h}$, les moyennes du lot $\mathrm{I}_{1}$ ne sont pas différentes de celles du lot $N(P>0,05$, tabl. 2$)$; cependant, les variances dans les 3 premières tranches (de $-9 \mathrm{~h}$ à $+9 \mathrm{~h}$ par rapport à Tf. max.) sont plus élevées pour le lot $I_{1}$ que pour le lot $N\left(P<0,05\right.$, tabl. 2). La courbe moyenne du lot $I_{2}$ suit une évolution parallèle à celle du lot $\mathrm{N}$ (fig. 4). Les moyennes du lot $\mathrm{I}_{2}$ sont supérieures à celles du lot $N$ pour chacune des tranches de $6 h(P<0,05$, tabl. 2). Les variances ne sont pas différentes $(P>0,05$, tabl. 2$)$.

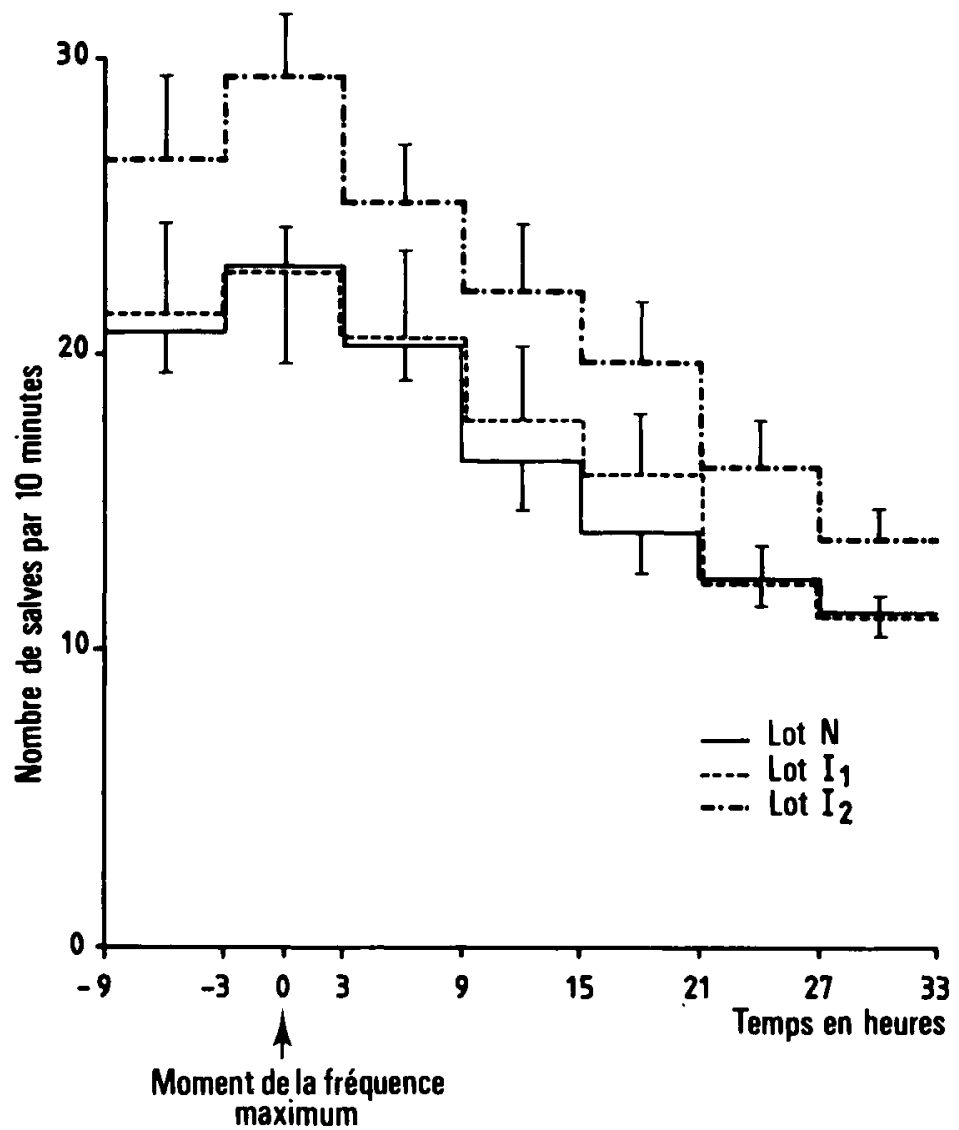

FIG. 4. - Evolution moyenne de la fréquence des salves de potentiels chez 3 lots de brebis. Lot $\mathrm{N}$ : œstrus naturel ; Lot $\mathrm{I}_{1}$ : œstrus inquit par les progestagènes ; Lot $\mathrm{l}_{2}$ : œstrus induit par les progestagènes avec une supplémentation de PMSG.

Abcisses : temps en heures par rapport au moment de la fréquence maximum ; Ordonnées : moyenne \pm sem du nombre de salves par $10 \mathrm{~min}$, déterminée pour chacun des 3 lots par tranches successives de $6 \mathrm{~h}$.

3. - Organisation de l'activité utérine. - Sur une même corne utérine, les activités EMG ne sont pas recueillies simultanément au niveau des différentes électrodes. Ainsi, la figure $\mathbf{5}$ montre les différents cas possibles dans l'ordre 
d'apparition des salves de potentiels : I'activité électrique est enregistrée successivement soit par les électrodes $A, B$ et $C$ dans le sens jonction utéro-tubaire (JUT)cervix (1) (propagation descendante), soit par les électrodes C, B et A dans le sens cervix-JUT (2) (propagation ascendante); d'autres successions sont également observées (3) (propagation non définie).

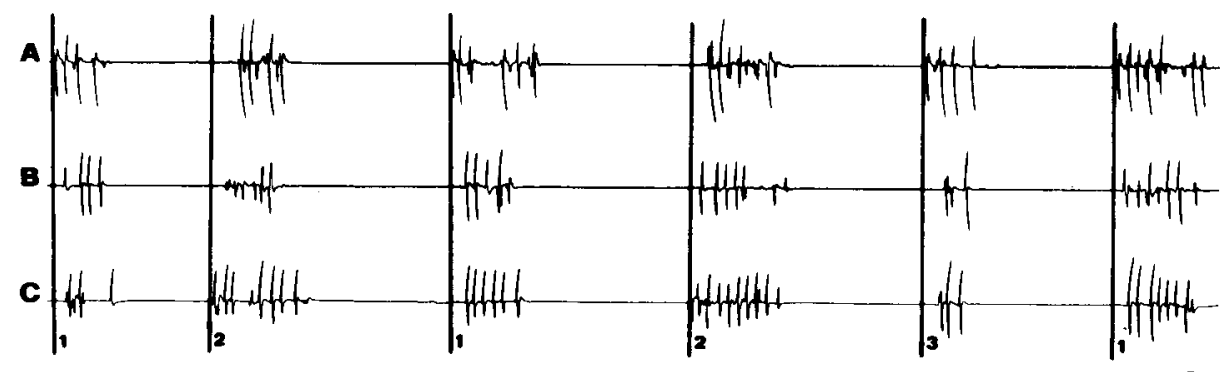

FIG. 5. - Enregistrement de l'activité électrique du myomètre en 3 points d'une même corne utérine au cours de l'cestrus.

A : extrémité de la corne utérine à proximité de la jonction utéro-tubaire (JUT) ; B : position intermédiaire ; $\mathrm{C}$ : extrémité cercivale de la corne utérine.

Un trait plein vertical passant par le début de la salve apparaissant la première, permet d'apprécier l'ordre d'apparition des salves.

1 : propagation dans le sens JUT-cervix ; 2 : propagation dans le sens cervix-JUT ; 3 : autres successions. Etalonnages : Amplitude : $1 \mathrm{mV}$; Temps : $5 \mathrm{~s}$.

Pour le lot $N$, nous ne pouvons définir de différences entre les pourcentages des 3 types de propagation à aucun des 5 moments qui sont analysés $(P>0,05$, Test de Friedman) (tabl. 3). Pour le lot $I_{1}$, les pourcentages des 3 types de propagaion ne sont pas différents jusqu'à $12 \mathrm{~h}$ après le début du pic de $\mathrm{LH}(\mathrm{P}>0,05)$. En revanche, 24 et $36 \mathrm{~h}$ après le début du pic de $\mathrm{LH}$, la propagation non définie est significativement supérieure aux autres $(P<0,05$, tests de Friedman et de Kramer sur la somme des rangs) (tabl. 3). Pour le lot $I_{2}$, comme pour le lot $N$, nous ne pouvons définir, à aucun moment, de différences significatives entre les 3 types de propagation $(P>0,05)$ (tabl. 3 ).

\section{Discussion.}

Nous savons que l'activité rythmique spontanée de l'utérus chez la brebis au cours de l'œestrus est le résultat de l'action des œestrogènes (Rousseau et Prud'homme, 1974). Le niveau d'œstradiol 17 $\beta$, principal œstrogène sécrété chez la brebis, est maximum $40 \mathrm{~h}$ avant le début de l'œestrus (Moore et al., 1969 ; Cox et al., 1971) et explique l'activité EMG rythmique recueillie au cours de l'œstrus des brebis des 3 lots. Les activités utérines maximales, au cours de l'œestrus naturel, sont réparties sur une période de $13 \mathrm{~h}$ de part et d'autre du début du pic de LH). Dans 50 \% des cas, ce maximum se situe pendant la décharge de LH. Les délais nécessaires aux cestrogènes pour agir sur différents effecteurs (complexe hypothalamo-hypophysaire et utérus) sont comparables. 
1. - Effet des progestagènes. - La fréquence des salves de potentiels est la même au cours de l'œstrus induit par les progestagènes seuls et au cours de l'œstrus naturel, ce qui est le résultat d'une production d'œestrogènes semblable dans les deux cas (Smith et Robinson, 1970). Nos résultats confirment ceux de

TABLEAU 3

Pourcentages, en fonction du temps, des propagations descendante, ascendante et non définie chez les 3 lots de brebis.

$\%$ des salves apparaissant dans le sens

\begin{tabular}{|c|c|c|}
\hline $\begin{array}{c}\text { JUT-cervix } \\
\text { (propagation } \\
\text { descendante) }\end{array}$ & $\begin{array}{l}\text { cervix-JUT } \\
\text { (propagation } \\
\text { ascendante) }\end{array}$ & $\begin{array}{c}\text { autres successions } \\
\text { (propagation non } \\
\text { définie) }\end{array}$ \\
\hline
\end{tabular}

Début du pic de LH (To)

\begin{tabular}{|c|c|c|c|c|c|}
\hline $\begin{array}{l}\text { Lot } N \\
(n=6)\end{array}$ & $\begin{array}{l}\bar{x} \pm \text { s.e.m. } \\
\text { Total des rangs }\end{array}$ & $44,7 \pm 10,4$ & $\frac{25,2 \pm 9,3}{9}$ & $30,1 \frac{ \pm}{12}$ & 5,8 \\
\hline $\begin{array}{l}\text { Lot I } 1 \\
(n=7)\end{array}$ & $\begin{array}{l}\bar{x} \pm \text { s.e.m. } \\
\text { Total des rangs }\end{array}$ & $31 \pm \frac{t}{14}$ & $\underset{14}{33,1 \pm 7,1}$ & $35,8 \pm$ & 6,6 \\
\hline $\begin{array}{l}\text { Lot } \mathrm{I}_{2} \\
(\mathrm{n}=2)\end{array}$ & $\begin{array}{l}\bar{x} \pm \text { s.e.m. } \\
\text { Total des rangs }\end{array}$ & $48,2 \pm$ & $\frac{21,3 \pm 3,6}{2}$ & $30,4 \frac{ \pm}{5}$ & 6,1 \\
\hline
\end{tabular}

$6 \mathrm{~h}$ après To

$\begin{array}{llccc}\text { Lot } \mathrm{N} & \overline{\mathrm{x}} \pm \text { s.e.m. } & 40,2 \pm 8,2 & 19,2 \pm 6,1 & 40,6 \pm 5,1 \\ \text { (n }=4) & \text { Total des rangs } & 9 & 5 & 10 \\ \text { Lot } \mathrm{I}_{1} & \overline{\mathrm{x}} \pm \text { s.e.m. } & 35,0 \pm 11,6 & 24,0 \pm 2,7 & 41,1 \pm 9,8 \\ \text { (n }=4) & \text { Total des rangs } & 10 & 5,5 & 8,5 \\ \text { Lot } \mathrm{I}_{2} & \overline{\mathrm{x}} \pm \text { s.e.m. } & 28,6 \pm 12,2 & 34,7 \pm 8,5 & 36,7 \pm 7,1 \\ (\mathrm{n}=5) & \text { Total des rangs } & 10 & 9 & 11\end{array}$

$12 \mathrm{~h}$ après To

\begin{tabular}{|c|c|c|c|c|}
\hline $\begin{array}{l}\text { Lot } N \\
(n=4)\end{array}$ & $\begin{array}{l}\bar{x} \pm \text { s.e.m. } \\
\text { Total des rangs }\end{array}$ & $41,5 \pm 12,5$ & $28,5 \pm 9,9$ & $30,0 \pm \frac{ \pm}{7}$ \\
\hline $\begin{array}{l}\text { Lot } I_{1} \\
(n=5)\end{array}$ & $\begin{array}{l}\bar{x} \pm \text { s.e.m. } \\
\text { Total des rangs }\end{array}$ & $37,8 \pm$ & $21,6 \pm \frac{ \pm}{7}+2$ & $40,4 \pm \frac{1}{13}$ \\
\hline $\begin{array}{l}\text { Lot } I_{2} \\
(n=5)\end{array}$ & $\begin{array}{l}\bar{x} \pm \text { s.e.m. } \\
\text { Total des rangs }\end{array}$ & $23,7 \underset{8}{ \pm}$ & $\begin{array}{c}34,1 \pm 7,3 \\
11\end{array}$ & $42,1 \pm$ \\
\hline
\end{tabular}

$24 \mathrm{~h}$ après To

\begin{tabular}{|c|c|c|c|c|c|c|}
\hline $\begin{array}{l}\text { Lot } N \\
(n=5)\end{array}$ & $\begin{array}{l}\bar{x} \pm \text { s.e.m. } \\
\text { Total des rangs }\end{array}$ & $31,0 \pm$ & 1,1 & $\underset{11}{34,2} \pm 6,0$ & $34,8 \pm$ & 6,2 \\
\hline $\begin{array}{l}\text { Lot } I_{1} \\
(n=3)\end{array}$ & $\begin{array}{l}\bar{x} \pm \text { s.e.m. } \\
\text { Total des rangs }\end{array}$ & $20,0 \pm \frac{ \pm}{3}$ & 1,9 & $28,6 \pm 3,9$ & $51,3 \pm \frac{ \pm}{9^{*}}$ & 4,1 \\
\hline $\begin{array}{l}\text { Lot } \mathrm{I}_{2} \\
\langle\mathrm{n}=4)\end{array}$ & $\begin{array}{l}\bar{x} \pm \text { s.e.m. } \\
\text { Total des rangs }\end{array}$ & $25,1 \pm \frac{}{6,5}$ & 7,1 & $\begin{array}{c}32,8 \pm 2,8 \\
6,5\end{array}$ & $42,3 \pm$ & 5,1 \\
\hline
\end{tabular}

\section{$36 \mathrm{~h}$ après To}

$\begin{array}{ll}\text { Lot } N & \bar{x} \pm \text { s.e.m. } \\ \text { (n }=4) & \text { Total des rangs } \\ \text { Lot } I_{1} & \bar{x} \pm \text { s.e.m. } \\ (n=3) & \text { Total des rangs } \\ \text { Lot } I_{2} & \bar{x} \pm \text { s.e.m. } \\ (n=2) & \text { Total des rangs }\end{array}$

$32,0 \pm 5,3$
$\begin{aligned} & 8,5 \\ & 13,3 \pm \\ & 4 \\ & 36,1 \underset{4,5}{ \pm} 8,3\end{aligned}$
$23,0 \pm 5,3$
$18,6 \pm 9,6$
5
$24,9 \pm 2,8$
2,5
$44,7 \pm \frac{ \pm}{9,5} 6,2$
$67,7 \pm 12,1$
$9^{*}$
$39,0 \pm 5,6$

* Statistiquement supérieur aux deux autres pourcentages $(P<0,05)$. 
Croker et Shelton (1973). En revanche, ils diffèrent de ceux de Hawk et Echternkamp (1973) qui observent que le traitement par les progestagènes réduit la fréquence des contractions. Mais les observations de ces auteurs sont faites pendant de courtes périodes (10 min) sur l'utérus extériorisé chez la brebis anesthésiée, alors que nous effectuons des enregistrements continus durant tout I'œstrus chez la brebis éveillée. Les différences de résultats sont vraisemblablement dues aux méthodes utilisées.

Cependant, dans l'œstrus induit, la période d'activité utérine maximale est retardée de $7 \mathrm{~h}$ par rapport à ce qui est observé dans l'œestrus naturel. Ceci explique qu'en moyenne, la fréquence des salves chute plus tôt dans le premier cas que dans le second.

Les progestagènes augmentent nettement la variabilité de la fréquence des salves entre animaux pendant une période de $18 \mathrm{~h}$ autour de la fréquence maximale. L'organisation de la motricité utérine est également modifiée. Au cours de l'œstrus naturel, la variabilité est telle qu'aucune propagation n'est privilégiée à aucun moment de l'œstrus. Cette variabilité peut être due au fait que les périodes d'une heure choisies pour cette analyse sont d'une durée insuffisante. II en résulte cependant que sous l'influence des progestagènes, le pourcentage de propagation non définie est prédominant 24 et $36 \mathrm{~h}$ après le début du pic de LH. Hawk et Echternkamp (1973) observent au contraire que la propagation ascendante prépondérante au cours de l'œstrus naturel est diminuée par le traitement progestatif. Leurs analyses ont été effectuées sur des périodes de $10 \mathrm{~min}$, c'est-à-dire plus courtes que celles que nous avons utilisées pour notre étude. II est donc difficile de comparer les 2 groupes de résultats.

2. - Effet de la PMSG. - Lorsqu'une administration de PMSG est associée aux progestagènes, des différences apparaissent par rapport à l'œestrus naturel. Les moments, par rapport à la décharge de LH, où l'activité utérine est maximale sont plus dispersés. La PMSG augmente la fréquence des salves; la décharge d'œstrogènes plus élevée dans ces conditions (Evans et Robinson, 1980) peut expliquer cette augmentation. Cependant, comme pour l'œstrus naturel, aucune différence significative n'est observée entre les pourcentages des 3 types de propagation à aucun moment de l'œstrus.

Ainsi, l'augmentation de la variabilité entre animaux observée pour la fréquence en début d'œestrus et la prépondérance de la propagation non définie observée en fin d'œstrus sous l'influence des progestagènes, n'apparaissent plus lorsque la PMSG est associée au traitement progestatif.

\section{Conclusion.}

La diminution de la fertilité observée chez la brebis, sous l'influence des progestagènes $(54,5 \%$ vs $72 \%$ ) (Colas et al., 1973) a été attribuée à des perturbations du transit du sperme (Quinlivan et Robinson, 1967, 1969 ; Hawk et Conley, 1971, 1972). L'ovulation a lieu en moyenne 24 h (entre 21 et 26 h) après le début du pic de LH (Cumming et al., 1973). Etant donné la durée du transit du sperme (de 4 à $8 \mathrm{~h}$ selon Dauzier et du Mesnil du Buisson, 1956), la période critique pour 
le transport des spermatozoïdes se situe dans les heures qui précèdent le moment de l'ovulation. La prépondérance de la propagation non définie, observée au cours de l'œstrus induit par les progestagènes seuls 24 et $26 \mathrm{~h}$ après To, c'est-àdire, au moment de l'ovulation et après celle-ci se manifeste trop tard pour avoir un effet sur le transit du sperme. En revanche, la fréquence des salves est variable entre les brebis au moment du maximum (c'est-à-dire, dans les $6 \mathrm{~h}$ qui suivent le début du pic de $\mathrm{LH}$ ). Si le transit du sperme dépend de la fréquence des salves, il est possible qu'il soit anormal pour les brebis dont la fréquence maximale est très en dessous de la moyenne. La PMSG, en augmentant celle-ci, préviendrait ces perturbations, ce qui expliquerait que, dans ces conditions, la fertilité ne soit pas modifiée par rapport à celle observée dans l'œestrus naturel (69\% vs $71,8 \%$ ) (Colas, 1975).

Reçu en décembre 1982. Accepté en septembre 1983.

\section{Références}

COLAS G., 1975. The use of progestagen SC 9880 as an aid for artificial insemination in ewes. Ann. Biol. anim. Bioch. Biophys., 15, 317-327.

COLAS G., THIMONIER J., COUROT M., ORTAVANT R., 1973. Fertilité, prolificité et fécondité pendant la saison sexuelle des brebis inséminées artificiellement après le traitement à l'acétate de fluorogestone. Ann. Zootech., 22, 441-451.

COX R. I., MATTNER P. E., THORBURN G. D., 1971. Changes in ovarian secretion of œstradiol $17 \beta$ around the cestrous in the sheep. J. Endocr., 49, 345-346.

CROCKER K. P., SHELTON J. N., 1973. Influence of stage of cycle, progestagen treatment and dose of cestrogen on uterine motility in the ewe. J. Reprod. Fert., 32, 521-524.

CUMMING I. A., BUCKMASTER J. M., BLOCKEY M. A. de B., GODING J. R., WINFIELD C. G., BAXTER R. W., 1973. Constancy of interval between luteinizing hormone release and ovulation in the ewe. Biol. Reprod., 9, 24-29.

DAUZIER L., du MESNIL du BUISSON F., 1956. Les spermatozoïdes dans l'appareil génital femelle. Proc. 2nd world Congr. Fertil. Steril., Naples, 1149-1159.

EVANS G., ROBINSON T. J., 1980. The control of fertility in sheep : endocrine and ovarian responses to progestagen-PMSG treatment in the breeding season and in anoestrus. J.agric. Sci. Camb., 94, 69-88.

HAWK H. W., CONLEY H. H., 1971. Loss of spermatozoa from the reproductive tract of the ewe and intensification of sperm «breakage " by progestagen. J. Reprod. Fert., 27, 339-347.

HAWK H. W., CONLEY H. H., 1972. Investigation of sperm transport failures in ewes administered synthetic progestagen. J. anim. Sci., 34, 609-613.

HAWK H. W., ECHTERNKAMP S. E., 1973. Uterine contractions in the ewe during progestagenregulated cestrus. J. Reprod. Fert., 34, 347-349.

MATTNER P. E., 1963. Spermatozoa in the genital tract of the ewe. III. The role of spermatozoa motility and of uterine contractions in transport of spermatozoa. Aust. J. biol. Sci., 16, 877884.

MOORE N. W., BARRET S., BROWN J. B., SCHINDLER I., SMITH M. A., SMYTH B., 1969. Oestrogen and progesterone content of ovarian vein blood of the ewe during the cestrous cycle. J. Endocr., 44, 55-62.

PELLETIER J., KANN G., DOLAIS J., ROSSELIN G., 1968. Dosage radioimmunologique de LH plasmatique chez le mouton. Mise au point de la technique de dosage. C. R. Acad. Sci. Paris, sér. D., 266, 2291-2294. 
QUINLIVAN T. D., ROBINSON T. J., 1967. The number of spermatozoa in the fallopian tubes of ewes at intervals after artificial insemination following withdrawal of SC 9880 impregnated intravaginal sponges, 177-194. In ROBINSON T. J., The control of the ovarian cycle in the sheep. Sydney Univ. Press.

QUINLIVAN T. D., ROBINSON T. J., 1969. Numbers of spermatozoa in the genital tract after artificial insemination of progestagen-treated ewes. J. Reprod. Fert., 19, 73-86.

ROUSSEAU J. P., PRUD'HOMME M. J., 1974. Etude électromyographique de la motricité de l'utérus chez la brebis. Action des hormones. Ann. Biol. anim. Bioch. Biophys., 14, 67-85.

SMITH J. F., ROBINSON T. J., 1970. The effet of exogenous progestagen on the levels of free œestrogen in the ovarian vein plasma of the ewe. J. Endocr., 48, 485-496. 